\title{
Image Analysis of Defocused Single-Molecule Images for Three-Dimensional Molecule Orientation Studies
}

\author{
Digambara Patra, Ingo Gregor, and Jörg Enderlein* \\ Institute for Biological Information Processing I, Forschungszentrum Jülich, D-52425 Jülich, Germany
}

Received: April 26, 2004; In Final Form: June 15, 2004

\begin{abstract}
An efficient algorithm for pattern matching has been developed based on least-squares analysis of fitting a discrete set of master patterns against measured images. This algorithm has been applied to determine threedimensional molecule orientations in defocused single-molecule images. The developed algorithm exploits the excellent agreement between electrodynamic calculations of single-molecule emission and experimentally measured images. The procedure is found to be reliable and simple and can be applied to any kind of pattern recognition where the patterns to be recognized are precisely known a priori. The procedure works well even for noisy and low-intensity signals as usually encountered in single-molecule experiments.
\end{abstract}

\section{Introduction}

Fluorescence spectroscopy of single molecules has become a routine technique for studying the physical and chemical behavior of individual molecules and has become an increasingly important tool for molecular biology (for a recent overview of the field see ref 1). A topic of special interest has always been the experimental determination of the spatial orientation of the molecule's absorption/emission dipole. The information about this orientation is important for several reasons. On one hand, photophysical parameters of single molecules, such as fluorescence lifetime and observable emission intensity, are often dependent on their orientation. On the other hand, singlemolecule orientation can itself be a probe for studying photophysics $^{2,3}$ or the structure of the embedding environment ${ }^{4,5}$ or for probing the orientation of labeled biomolecules. ${ }^{6-10}$ In the past, several techniques have been proposed for determining single-molecule orientations by fluorescence imaging. The core idea of these approaches is to obtain information about the angular distribution of a single-molecule's fluorescence emission by "deteriorating" the image of the molecule either by introduction of aberration, ${ }^{1-14}$ by defined image defocusing, ${ }^{15-17}$ or by imaging of the collected fluorescence light with a Bertrand lens. ${ }^{18}$ In all cases, the intensity distribution of the blurred image contains information about the molecule's emission-dipole orientation. The defocusing method was proposed in refs 15 and 16 and experimentally demonstrated for an immersion mirror objective used for imaging within a cryostat at low temperature. In ref 17 , this concept was applied to image surface-bound molecules using a conventional CCD-imaging epi-fluorescence microscope with laser wide-field illumination. This method is easy to implement and allows for fast screening of single-molecule orientations, without the necessity of scanning, excitation light modulation, or multiple channel detection. Thus, it is useful for applications where three-dimensional orientations of single molecules constitute an important measurement parameter. However, for analyzing large numbers of molecule orientations, it was necessary to develop efficient

* To whom correspondence should be addressed. Fax: +49-2461 614216. E-mail: j.enderlein@fz-juelich.de. pattern recognition algorithms that can localize single-molecule patterns within an image and determine their three-dimensional orientation even under noisy low-signal conditions. The description of such an algorithm and the demonstration of its performance is the aim of the present paper.

\section{Theory}

Image Analysis. Let $x_{j k}$ be the intensity value of the pixel with coordinates $(j, k)$ in the original image. The task is to identify and localize pre-defined patterns within the image. Thus, the algorithm has to compare all possible subregions of the image with a discrete set of $R$ predefined patterns $p_{j k}^{(r)}$ which shall have the size $(2 L+1) \times(2 L+1)$ (with $1 \leq r \leq R$ counting the patterns and $-L \leq j \leq L$ and $-L \leq k \leq L$ counting the pixels of the patterns). Additionally, a uniform background pattern $b$ may be superimposed and the pattern-matching may be restricted to nonrectangular subareas defined by the support matrixes $s_{j k}^{(r)}$ of the same size as the patterns but having values 1 and 0 only. Furthermore, it is assumed that, within any given subarea of the image, only one of the $R$ patterns can be present. Pattern comparison is done in the following way. The algorithm fits, by a least-squares method, each of the $R$ patterns (plus a flat background) to all possible subareas of the image. For every subarea, the algorithm chooses the pattern yielding minimal least-squares error to be the most likely pattern present in the given subarea. Thus, for any given subarea of the image $x$ with pixels $m-L \leq j \leq m+L$ and $n-L \leq k \leq n+L$, the algorithm tries to find $2 R$ parameters $c_{n m}^{(r)}$ and $d_{n m}^{(r)}$ so that the least-squares errors $e_{m n}^{(r)}$

$$
e_{m n}^{(r)}=\sum_{j=-L k=-L}^{L} \sum_{j k}^{L} s_{j k+j, k}\left(x_{m n}-c_{m k}^{(r)} p_{j k}^{(r)}-d_{m n}^{(r)} b_{j k}\right)^{2}
$$

are minimized. Subsequently, it is assumed that the patterns $p_{j k}^{(r)}$ as well as the background pattern $b_{j k}$ are all squarenormalized so that 


$$
\left.\sum_{j=-L k=-L}^{L} \sum_{j k}^{L} s_{j k}^{(r)}\right)^{2}=1 \text { and } \sum_{j=-L k=-L}^{L} \sum_{j k}^{L} s_{j k}\left(b_{j k}\right)^{2}=1
$$

By differentiating with respect to the coefficients $c_{m n}^{(r)}$ and $d_{m n}^{(r)}$, these conditions lead to the $2 R$ equations

$$
\begin{gathered}
\left.\frac{\partial e_{m n}^{(r)}}{\partial c_{m n}^{(r)}}=\sum_{j=-L k=-L}^{L} \sum_{j k}^{L} s_{j+j, n+k}-c_{m n}^{(r)} p_{j k}^{(r)}-d_{m n}^{(r)} b_{j k}\right) p_{j k}^{(r)}=0 \\
\frac{\partial e_{m n}^{(r)}}{\partial b_{m n}^{(r)}}=\sum_{j=-L k=-L}^{L} \sum_{j k}^{L} s_{j k}\left(x_{m+j, n+k}-c_{m n}^{(r)} p_{j k}^{(r)}-d_{m n}^{(r)} b_{j k}\right) b_{j k}=0
\end{gathered}
$$

with $1 \leq r \leq R$. Introducing the abbreviations

$$
\begin{gathered}
X_{m n} \equiv \sum_{j=-L k=-L}^{L} \sum_{j k}^{L} s_{j k} x_{m+j, n+k} b_{j k} \\
Q_{m n}^{(r)} \equiv \sum_{j=-L k=-L}^{L} \sum_{j k}^{L} s_{m+j, n+k} p_{j k}^{(r)} \\
P^{(r)} \equiv \sum_{j=-L k=-L}^{L} \sum_{j k}^{L} p_{j k}^{(r)} b_{j k}
\end{gathered}
$$

the two equations can be rewritten in matrix form as

$$
\left(\begin{array}{ll}
1 & P^{(r)} \\
P^{(r)} & 1
\end{array}\right)\left(\begin{array}{l}
c_{m n}^{(r)} \\
b_{m n}^{(r)}
\end{array}\right)=\left(\begin{array}{l}
Q_{m n}^{(r)} \\
X_{m n}
\end{array}\right)
$$

with the explicit solution

$$
\begin{aligned}
\left(\begin{array}{l}
c_{m n}^{(r)} \\
b_{m n}^{(r)}
\end{array}\right)=\left(\begin{array}{ll}
1 & P^{(r)} \\
P^{(r)} & 1
\end{array}\right)^{-1}\left(\begin{array}{l}
Q_{m n}^{(r)} \\
X_{m n}
\end{array}\right)= & \frac{1}{\sqrt{1-\left(P^{(r)}\right)^{2}}}\left(\begin{array}{ll}
1 & -P^{(r)} \\
-P^{(r)} & 1
\end{array}\right)\left(\begin{array}{l}
Q_{m n}^{(r)} \\
X_{m n}
\end{array}\right)
\end{aligned}
$$

The square normalization of the patterns, together with the condition that all pixel values of the patterns are non-negative (and at least one pixel value positive), ensures that $0<P^{(r)}<$ 1 and that the solution is well-defined and real. With the coefficients $c_{m n}^{(r)}$ and $b_{m n}^{(r)}$ explicitly known, the $R$ errors $e_{m n}^{(r)}$ are calculated as

$$
e_{m n}^{(r)}=\left(X^{2}\right)_{m n}-\frac{\left(Q_{m n}^{(r)}\right)^{2}+\left(X_{m n}\right)^{2}-2 P^{(r)} Q_{m n}^{(r)} X_{m n}}{\sqrt{1-\left(P^{(r)}\right)^{2}}}
$$

where the additional abbreviation

$$
\left(X^{2}\right)_{m n} \equiv \sum_{j=-L k=-L}^{L} \sum_{j k}^{L} x_{m+j, n+k}^{2}
$$

was introduced. The beauty of eqs $6-8$ is that they involve only two-dimensional convolutions of the image $x$ with the patterns $p^{(r)}$ and $b$, which can be calculated efficiently by using fast Fourier transforms (see Appendix). Thus, the flow of the image analysis algorithm is summarized as follows:

1. Calculate the $R+1$ two-dimensional convolutions of the image $x$ with the $R$ patterns $p^{(r)}$ and the background pattern $b$; see eq 4.

2. Calculate the $2 R$ "coefficient images" $c^{(r)}$ and $d^{(r)}$ according to eq 6 .

3. Calculate the $R$ "error images" $e^{(r)}$ according to eq 7 , involving the calculation of the "squared image" $X^{2}$.

4. For all positions $(m, n)$, the pattern with minimum error $e_{m n}^{(r)}$ is chosen as the most likely pattern for that position. Thus, two new images $\tilde{c}$ and $\tilde{e}$ are generated with values $\tilde{c}_{m n}$ and $\tilde{e}_{m n}$ composed by those values $c_{m n}^{(r)}$ and $e_{m n}^{(r)}$ having minimum $e_{m n}^{(r)}$.

5. Finally, pattern positions are localized by asking for positions $(m, n)$ where the ratio $\tilde{c}_{m n} / \sqrt{\tilde{e}_{m n}}$ exceeds some predefined value $\kappa$. We have found that an appropriate value for $\kappa$ yielding excellent pattern recognition is close to 1 .

The last step is the only one containing an arbitrary parameter (the threshold value $\kappa$ ).

Electrodynamics of Singe-Molecule Imaging. The electrodynamics and optics of focused and defocused imaging of single fluorescent molecules was described in detail in a recent paper. ${ }^{17}$ Within the framework of classical electrodynamics, fluorescing molecules can be considered as ideal electric dipole emitters. When placed within a dielectrically homogeneous medium, such emitters exhibit the classical angular distribution of radiation proportional to $\sin ^{2} \theta$, where $\theta$ is the angle between the direction of observation and the dipole's axis. However, when placed close to an interface separating two media with different refractive indices, that angular distribution of radiation changes dramatically due to the self-interaction of the emitting dipole with its back-reflected electromagnetic field. ${ }^{19-21}$ Consider a molecule embedded in glass a distance $z$ away from the glass/ air interface. The general geometry of imaging such a molecule is depicted in Figure 1. Neglecting any constant factors, the angular distribution of the electric-field $\mathbf{E}(\eta, \psi)$ connected with

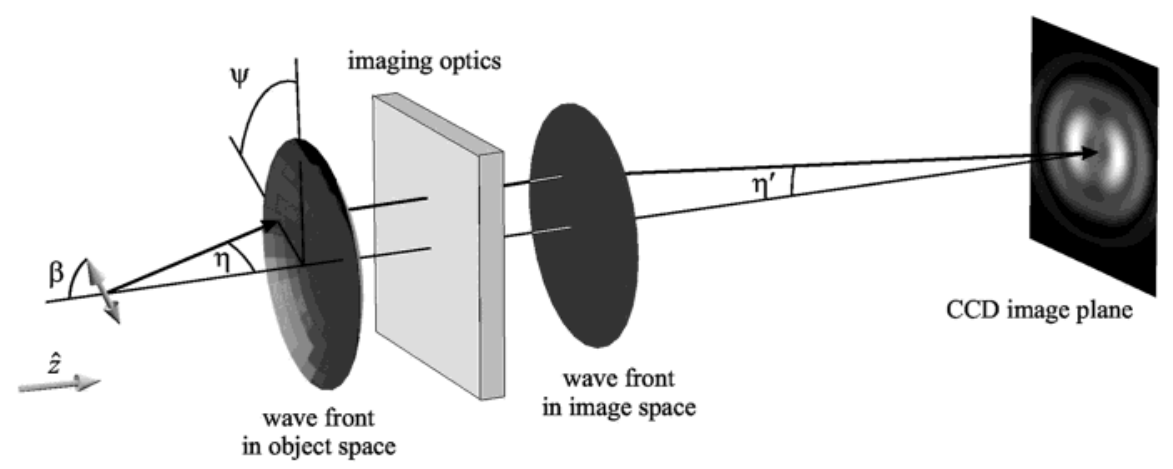

Figure 1. General geometry of defocused imaging. A fluorescing molecule (oscillating electric dipole) emits light that is collected by the imaging optics and imaged onto a CCD chip. With slight movement of the optics toward the molecule (negative $z$-direction in the figure), the image can be defocused to yield characteristic interference patterns that contain information about the three-dimensional orientation of the molecule. 
light radiation into the glass along direction $\hat{\mathbf{k}}=\{\sin \eta \cos \psi$, $\sin \eta \sin \psi, \cos \eta\}$ and solid angle $\sin \eta \mathrm{d} \eta \mathrm{d} \psi$ is given by (see, e.g., refs 17 and 21)

$$
\begin{aligned}
\mathbf{E}(\eta, \psi)=\mathbf{e}_{p}\left[\cos \beta E_{p}^{\perp}(\eta)+\right. & \\
& \left.\sin \beta E_{p}^{\prime \prime}(\eta) \cos \psi\right]+\mathbf{e}_{s} \sin \beta E_{s}^{\prime \prime}(\eta) \sin \psi
\end{aligned}
$$

with

$$
\begin{gathered}
E_{p}^{\perp}(\eta)=\sin \eta\left[\mathrm{e}^{-i n z \cos \eta}+R_{p}(\eta) \mathrm{e}^{i n z \cos \eta}\right] \\
E_{p, s}^{\|}(\eta)=\cos \eta\left[\mathrm{e}^{-i n z \cos \eta} \mp R_{p, s}(\eta) e^{i n z \cos \eta}\right]
\end{gathered}
$$

where $\beta$ is the angle between the molecule's emission dipole axis and the optical axis (being perpendicular to the interface); $\mathbf{e}_{p}$ and $\mathbf{e}_{s}$ are orthogonal unit vectors perpendicular to the radiation direction $\hat{\mathbf{k}}$ and with $\mathbf{e}_{s}$ oriented perpendicularly to the optical axis; $n$ denotes the refractive index of glass (that of air is assumed to be 1 ); and the $R_{p, s}(\eta)$ are Fresnel's reflection coefficients $^{22}$ for plane $p$ - and $s$-waves at the glass/air interface reflected along direction $\hat{\mathbf{k}}$ into the glass. Without restriction of generality it was assumed that the molecule's emission dipole lies within the plane $\psi=0$. The minus sign in the second of eq 10 refers to $p$-waves, the plus sign to $s$-waves. After imaging the radiation through an aplanatic imaging optics onto the CCD camera, the electric and magnetic fields on the surface of the CCD chip are given by ${ }^{17,23-25}$

$$
\left\{\begin{array}{l}
E_{j} \\
B_{j}
\end{array}\right\}=\int_{0}^{\eta^{\prime} \max } \mathrm{d} \eta^{\prime} \sin \eta^{\prime} \sqrt{\frac{\cos \eta^{\prime}}{n \cos \eta}}\left\{\begin{array}{l}
e_{j} \\
b_{j}
\end{array}\right\} \exp [i k \delta z \cos \eta]
$$

and

$$
\begin{aligned}
& \left\{\begin{array}{l}
e_{x} \\
e_{y}
\end{array}\right\}=\frac{i \sin \beta}{2} \times \\
& \left\{\begin{array}{l}
\cos \eta^{\prime}\left(J_{0}-J_{2} \cos 2 \psi\right) E_{p}^{\|}+\left(J_{0}+J_{2} \cos 2 \psi\right) E_{s}^{\|} \\
-\cos \eta^{\prime} J_{2} \sin 2 \psi E_{p}^{\|}+J_{2} \sin 2 \psi E_{s}^{\|}
\end{array}\right\}+ \\
& i \cos \beta \cos \eta^{\prime} J_{1} E_{p}^{\perp}\left\{\begin{array}{l}
\cos \psi \\
\sin \psi
\end{array}\right\}
\end{aligned}
$$

$$
\begin{aligned}
&\left\{\begin{array}{l}
b_{x} \\
b_{y}
\end{array}\right\}=\frac{i n^{\prime} \sin \beta}{2} \times \\
&\left\{\begin{array}{l}
-\cos \eta^{\prime} J_{2} \sin 2 \psi E_{s}^{\|}+J_{2} \sin 2 \psi E_{p}^{\|} \\
\cos \eta^{\prime}\left(J_{0}+J_{2} \cos 2 \psi\right) E_{s}^{\|}+\left(J_{0}-J_{2} \cos 2 \psi\right) E_{p}^{\|}
\end{array}\right\}+ \\
& i \cos \beta n^{\prime} J_{1} E_{p}^{\perp}\left\{\begin{array}{c}
-\sin \psi \\
\cos \psi
\end{array}\right\}
\end{aligned}
$$

where the $J_{0,1,2}$ denotes Bessel functions of the first kind ${ }^{26}$ with functional argument $k^{\prime} \rho^{\prime} \sin \eta^{\prime}$; the $k, k^{\prime}$ are the wave vectors in glass and air, respectively. The angles $\eta^{\prime}$ and $\eta$ are connected through the magnification $M$ via Abbe's sine condition (aplantic optics), $M \sin \eta^{\prime}=n \sin \eta$. The root factor in eq 11 ensures energy conservation during imaging and $\delta z$ is the value of image defocusing (how much the imaging objective is moved out of focus toward the sample). Finally, the position-dependent light intensity on the CCD chip is given by the z-component of the Poynting vector

$$
S=(c / 8 \pi) \mathbf{e}_{z} \cdot\left(\mathbf{E} \times \mathbf{B}^{*}\right)
$$

\section{Experiment}

The measurement system consisted of an inverted microscope (IX70, Olympus) equipped with $1.2 \mathrm{NA}, 100 \times$ plan fluorite

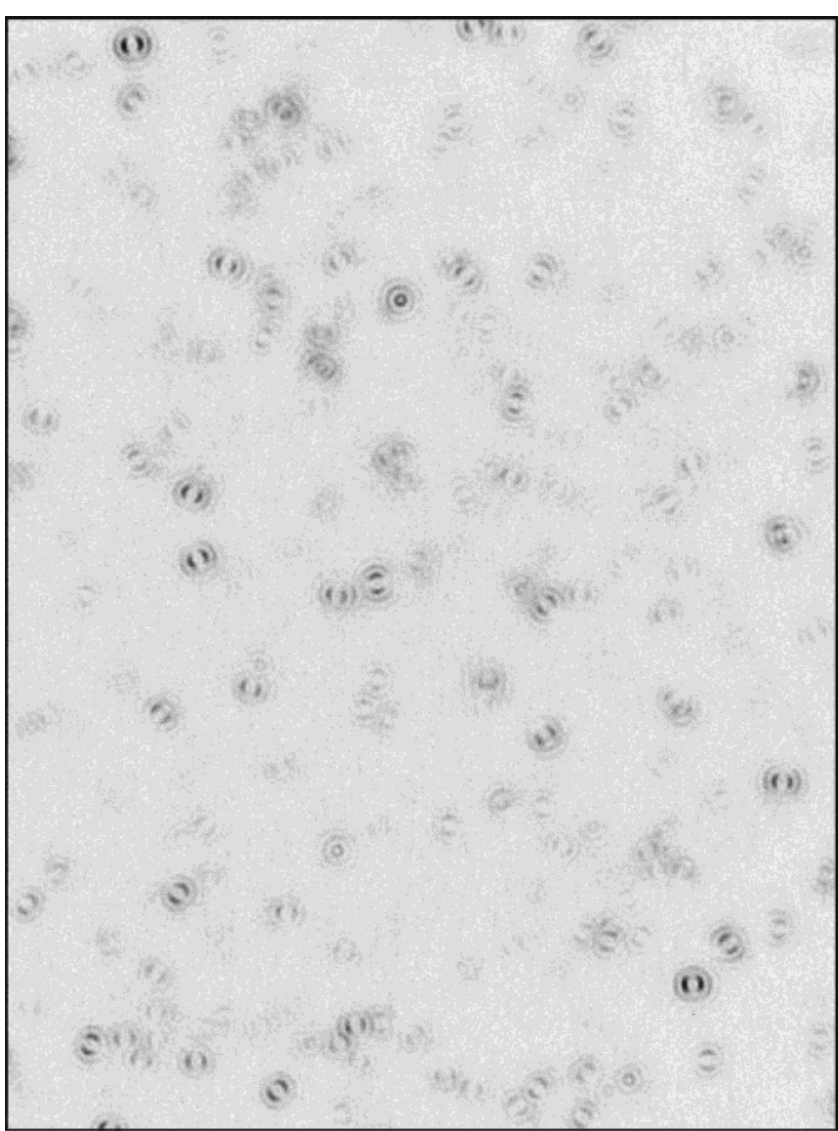

Figure 2. Measured defocused image of Cy5 molecules embedded in poly(vinyl alcohol) on glass near the air/polymer interface. Defocusing was achieved by moving the objective $1 \mu \mathrm{m}$ toward the sample.

apochromat oil immersion objective and a high-sensitive Peltiercooled CCD camera (CoolSnap HQ, Roper Scientific). Widefield illumination for fluorescence excitation was done with 37$\mathrm{mW}$ light of an Ar/Kr-ion laser (Stabilite 2018, Spectra-Physics) at $647.1 \mathrm{~nm}$, using an additional excitation filter (647NB4) within the excitation path. Excitation light was conducted to the objective using a multimode glass fiber. After passing the multimode fiber, the excitation light was nearly circularly polarized. A dichroic mirror (650DLRP, Omega Optical) reflected the excitation light into the back focal plane of the objective (Köhler illumination mode) for achieving homogeneous wide-field illumination of the sample. Fluorescence was collected by the same objective and was passed through the dichroic mirror and an additional emission filter (690DF40, Omega Optical) for background suppression (Raman/Rayleigh scattering). The CCD camera was positioned exactly at the image plane of the microscope's side port; the size of one CCD pixel is $6.45 \times 6.45 \mu \mathrm{m}^{2}$. The knowledge of this value allows direct conversion of pixel position into length and thus direct comparison between measured and calculated patterns. Axial positioning of the objective was performed with a piezoelectric transducer (PiFoc P-721-20, Physik Instrumente) with subnanometer resolution. The studied sample consisted of standard microscope cover slides (Menzel) of $170-\mu \mathrm{m}$ thickness, on top of which $100 \mu \mathrm{L}$ of a $10^{-9} \mathrm{M}$ solution of the cyanine dye Cy5 (Amersham) in bidistilled water containing 1\% wt/v poly(vinyl alcohol) (CLARIANT GmbH) was spin-coated and dried, yielding a sparse distribution of single Cy5 molecules within the polymer matrix. Glass and poly(vinyl alcohol) have a matching refractive index of ca. 1.52. Images of the sample were taken with an exposure time of 20 s. Image focusing/ 

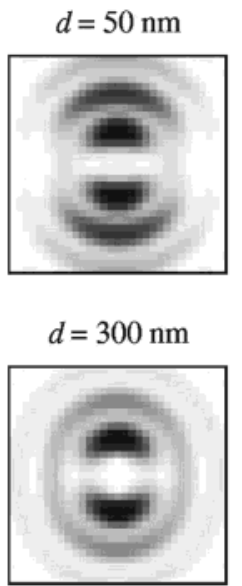

$d=100 \mathrm{~nm}$

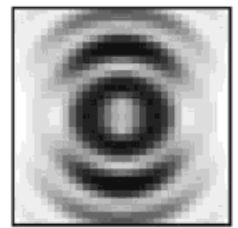

$d=350 \mathrm{~nm}$

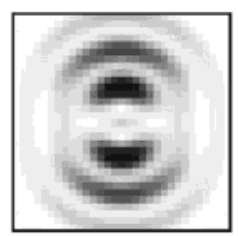

$d=150 \mathrm{~nm}$

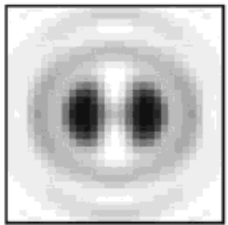

$d=400 \mathrm{~nm}$

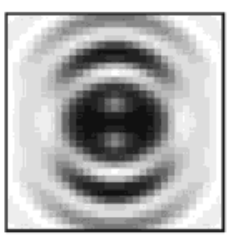

$d=200 \mathrm{~nm}$

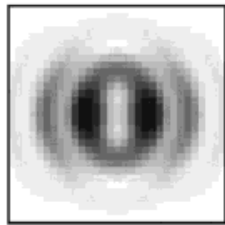

$d=450 \mathrm{~nm}$

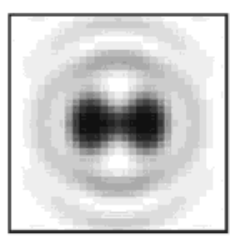

$d=250 \mathrm{~nm}$

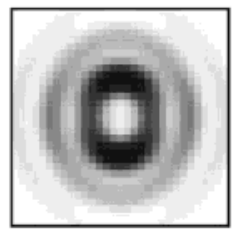

$d=500 \mathrm{~nm}$

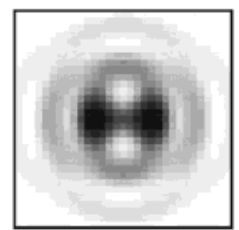

Figure 3. Calculated defocused images for a fixed defocusing value of $1.2 \mu \mathrm{m}$ with varying polymer film thickness. Above every pattern, value $d$ of the film thickness is indicated. All other parameters used in the calculations are the same as those in the experiment.
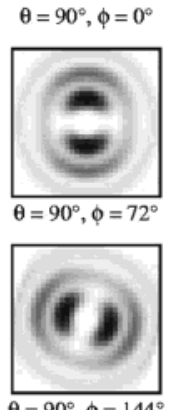

$\theta=90^{\circ}, \phi=144^{\circ}$
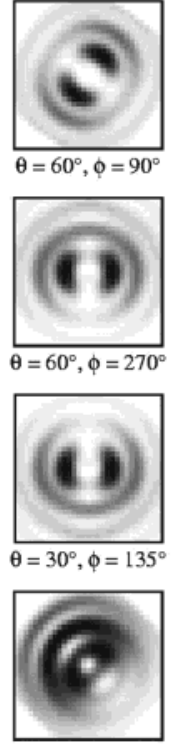
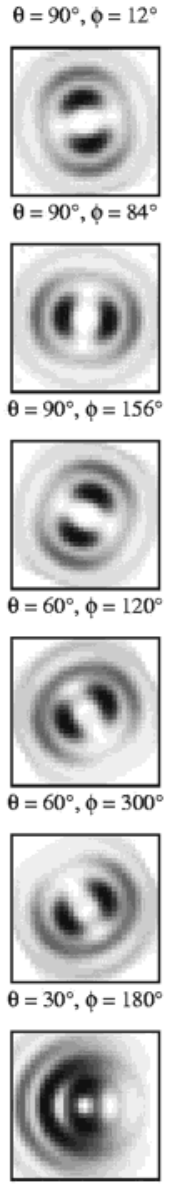
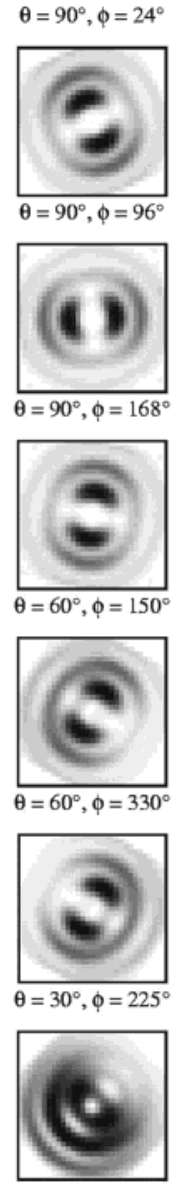
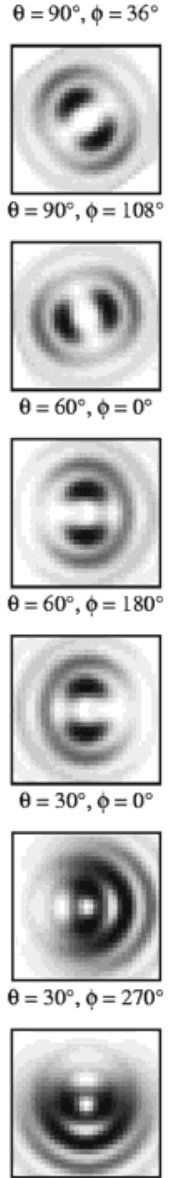
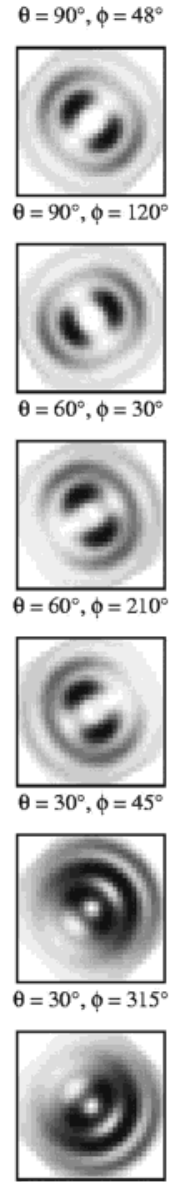
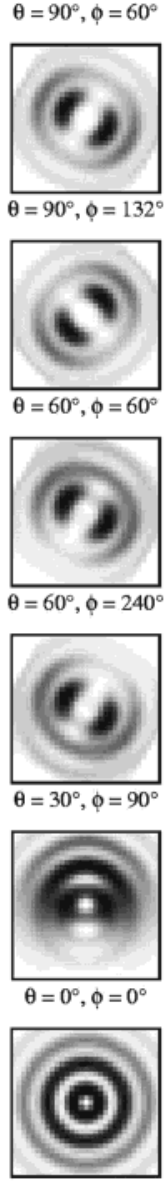

Figure 4. Calculated defocused images for 36 different orientations of an electric dipole emitter. Above every pattern, inclination angle $\theta$ between dipole and optical axis as well as angle $\phi$ of in-plane dipole orientation are indicated. All parameters used in the calculations are the same as those in the experiment.

defocusing was adjusted by moving the objective with the piezoelectric transducer.

\section{Results}

Figure 2 shows the complete CCD image of a defocused measurement, displaying the patterns of roughly a hundred molecules. The defocusing value for that image was $1.00 \pm$ $0.25 \mu \mathrm{m}$; i.e., the objective was first set to sharp focus and then moved toward the sample by an amount of $1 \mu \mathrm{m}$. The uncertainty in the absolute value of defocusing is caused by the uncertainty to find the exact position of sharp focus. Furthermore, the thickness of the polymer film between the molecules and the polymer/air interface is not exactly known. Fortunately, it occurs that the precise shape of the defocused patterns of single molecules with the dipole axis parallel to the air/polymer interface (in-plane dipole orientation) is very sensitive to both defocusing value and film thickness, which allows, by comparison between measurement and theoretical calculation, determination of both values simultaneously. For example, Figure 3 displays defocused patterns for varying values 


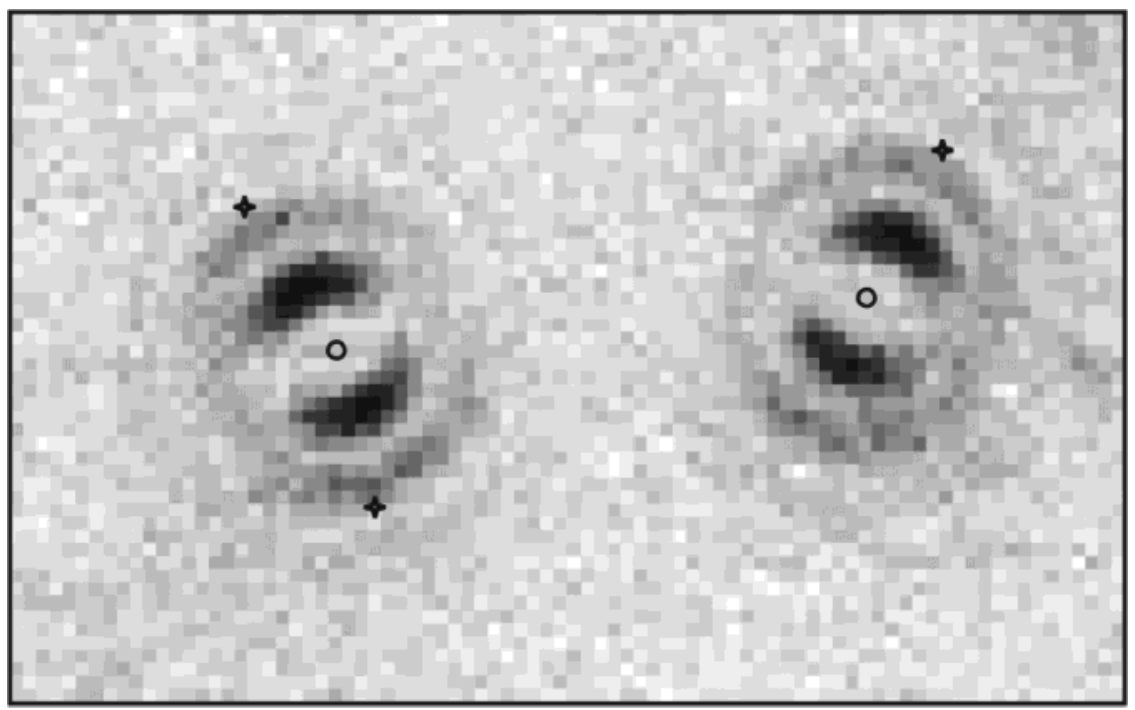

Figure 5. Example of correct and misidentified molecule positions (stars). Beside the exact position (circles, center of patterns), sometimes the algorithm identifies one-half of the bilaterally symmetric pattern as an image of a molecule with high inclination angle (stars, off-center positions).

of polymer film thickness (at a fixed defocusing value of 1.2 $\mu \mathrm{m})$, showing the dramatic changes in pattern shape with varying film thickness. It occurred that a defocusing value of $1.2 \mu \mathrm{m}$ and a film thickness value of $300 \mathrm{~nm}$ are matching the observed patterns best (compare Figure 3 with Figure 2). Moreover, because all molecules with in-plane dipole orientation display the same interference patterns in Figure 2, they have to be all located at the same distance to the air/polymer interface, assumedly adsorbed at the polymer/glass interface.

Fluorescence intensity of the molecules varies significantly from molecule to molecule, making it rather difficult to apply conventional image analysis methods which are based on intensity discrimination. To automatically identify single molecules and to determine their orientations, 36 master patterns of size $31 \times 31$ pixel for 36 different possible orientations were calculated (see Figure 4), giving the $p_{j k}^{(m)}$ of eq 1 . The defocused patterns are not very sensitive to the inclination of the dipole axis toward the optical axis. The bilaterally symmetric two-lobed images convert into circular ringed interference patterns only at large inclination angles. Thus, patterns at only four different inclination angles, $\theta=0,30,60$, and $90^{\circ}$, were computed. This shows also the limitation of the defocused imaging method for determination of dipole orientation: The precision of in-plane dipole orientation is much higher than that for out-of-plane inclination. A uniform background pattern $b_{j k}$ was assumed; the support matrix $s_{j k}$ was chosen to be a disk of 15 pixel radius. As the criterion for a positive match between a master pattern and a molecule image, the relation $\tilde{c}_{m n}>0.8$ $\sqrt{\tilde{e}_{m n}}$ was used (see step five of the algorithm in the first part of the Theory section). The center coordinates of such identified regions were taken as the center coordinates of identified molecules. However, it occurred that such a molecule localization method often generates spurious molecule positions besides the correct position: the typical error is that the algorithm identifies one-half of a bilaterally symmetric pattern of an inplane molecule as the image of a molecule with high inclination angle (see Figure 5). To avoid this, the matrix $\tilde{c}_{m n}$ was smoothed (convoluted with a uniform disk of 7 pixel radius) to yield $\tilde{c}_{m n}^{*}$, and only after that the criterion $\tilde{c}_{m n}^{*}>0.6 \sqrt{\tilde{e}_{m n}}$ was used for molecule localization. The algorithm identified 108 molecules, and the found patterns are displayed in Figure 6. Notice the excellent agreement between theory and observed patterns, demonstrating nicely that single fluorescing molecules behave

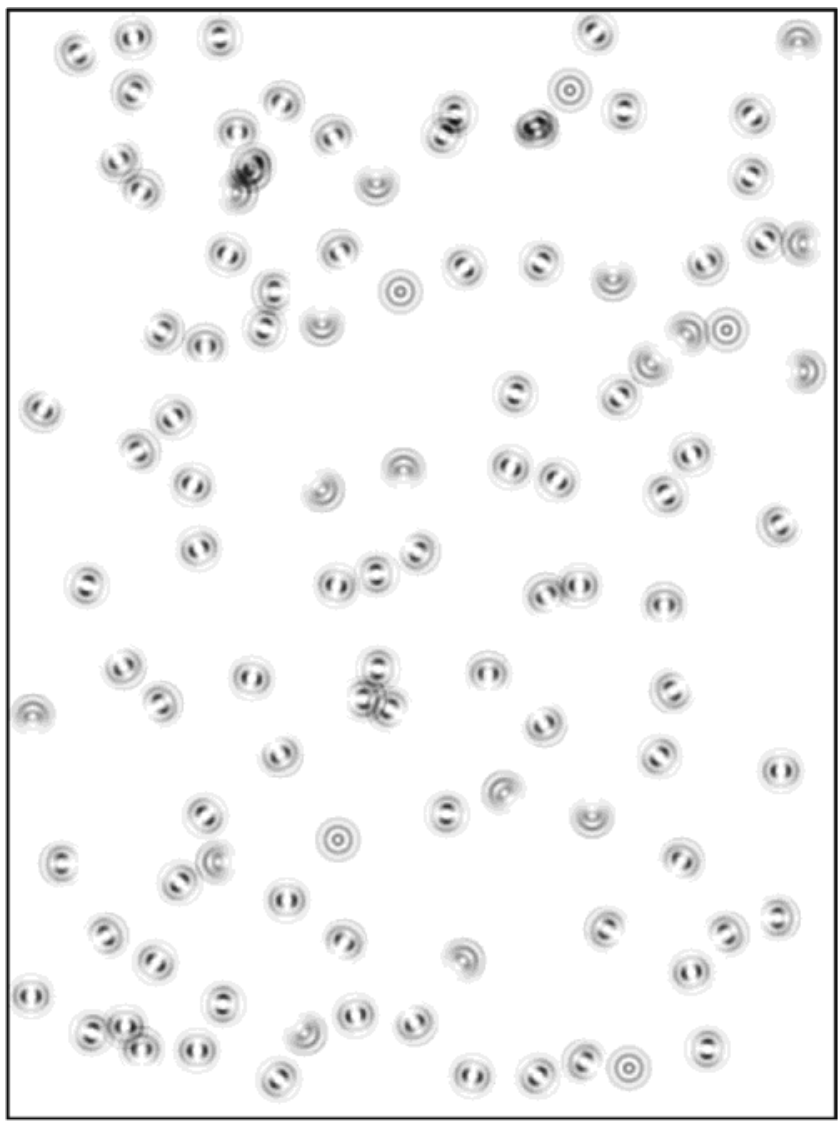

Figure 6. Composite image displaying all identified molecules and their attributed patterns.

indeed as ideal classical dipole emitters of light. As can be seen, the algorithm works quite well for sufficiently isolated molecule patterns, even at low signal strength. It is feasible to safely identify inclination angles with ca. $30^{\circ}$ resolution, and in-plane orientations with at least $15^{\circ}$ resolution. The algorithm has problems to localize molecules and to correctly identify their orientation if there is too much overlap between neighboring molecule patterns. This is easy to understand when taking into account that the algorithm tries to find the best match between a given image region and a discreet set of master patterns. Image regions with overlapping patterns that are not similar to any of 
the master patterns will likely produce senseless results. But this will probably be a problem for any image processing algorithm.

\section{Conclusion}

We have presented an efficient pattern matching algorithm based on a least-squares analysis of fitting a discrete set of master patterns against measured fluorescence images of single molecules. We applied the algorithm to defocused images of single fluorescing molecules, whose shapes depend strongly on the three-dimensional orientation of the molecule. The algorithm worked well, even for the low-intensity signals as experienced when imaging single molecules with defocused optics. It should be noticed that the algorithm is extremely sensitive to an exact correspondence between the master patterns and the measured patterns: using master patterns that only slightly deviate from the measured ones quickly deteriorates the recognition quality of the algorithm. Thus, the presented algorithm is especially useful for pattern recognition problems where the patterns to be recognized are precisely known a priori. In the case of defocused imaging of single molecules, this is indeed the case due to the excellent agreement between electrodynamic and optical theory and measurement.

Acknowledgment. We thank Martin Böhmer for his technical support, and we are grateful to Felix Koberling for lending us the 1.2 N.A. oil immersion objective. We are much obliged to Benjamin Kaupp for his generous support of our work. Financial support by the Deutsche Forschungsgemeinschaft (Grant EN 297/7), and the Humboldt Foundation is gratefully acknowledged.

\section{Appendix}

Convolution of an image $x$ with pixel values $x_{j k}$ and pixel range $1 \leq j \leq J, 1 \leq k \leq K$, with a smaller pattern $p$ with pixel values $p_{j k}$ and pixel range $-L \leq j, k \leq L(2 L+1<J, K)$, can be efficiently computed using fast Fourier transforms. The convolution $q$ is defined by

$$
q_{m n}=\sum_{j=-L k=-L}^{L} \sum_{m+j, n+k}^{L} x_{j k}
$$

By introducing the new field $p_{j k}^{*}$ with elements

$$
\begin{aligned}
& p_{j k}^{*}= \\
& \begin{cases}p_{j-L-1, k-L-1} & -L \leq j-L-1 \leq L \wedge-L \leq k-L-1 \leq L \\
0 & |j-L-1|>L \vee|k-L-1|>L\end{cases}
\end{aligned}
$$

and the new field $x_{j k}^{*}$ with cyclic elements

$$
x_{j k}^{*}=x_{[(j-L-2) \bmod J]+1,[(k-L-2) \bmod K]+1}
$$

this convolution can be rewritten as

$$
q_{m n}=\sum_{j=1}^{J} \sum_{k=1}^{K} x_{m+j, n+k}^{*} p_{j k}^{*}
$$

Next, the images $x^{*}$ and $p^{*}$ are represented by their two- dimensional Fourier transforms $\tilde{x}^{*}$ and $\tilde{p}^{*}$ as

$$
\begin{aligned}
x_{j k}^{*} & =\frac{1}{J K_{r}} \sum^{J} \sum_{s=1}^{K} \tilde{x}_{r s}^{*} \exp \left[2 \pi i\left(\frac{r j}{J}+\frac{s k}{K}\right)\right] \\
p_{j k}^{*} & =\frac{1}{J K} \sum_{r=1}^{J} \sum_{s=1}^{K} \tilde{p}_{r s}^{*} \exp \left[2 \pi i\left(\frac{r j}{J}+\frac{s k}{K}\right)\right]
\end{aligned}
$$

Inserting the last equations into eq A1 yields

$$
\begin{aligned}
q_{m n}= & \frac{1}{(J K)^{2}} \sum_{j=1}^{J} \sum_{k=1}^{K} \sum_{r=1}^{J} \sum_{s=1}^{K} \sum_{u=1}^{J} \sum_{v=1}^{K} \tilde{x}_{r s}^{*} \tilde{p}_{u v}^{*} \times \\
& \quad \exp \left[2 \pi i\left(\frac{r m+(u+r) j}{J}+\frac{s n+(v+s) k}{K}\right)\right] \\
= & \frac{1}{J K} \sum_{r=1}^{J} \sum_{s=1}^{K} \tilde{x}_{r s}^{*} \tilde{p}_{-r,-s}^{*} \exp \left[2 \pi i\left(\frac{r m}{J}+\frac{s n}{K}\right)\right]
\end{aligned}
$$

showing that the Fourier transform $\tilde{q}_{m n}$ of the convolution has the simple form

$$
\tilde{q}_{r s}=\tilde{x}_{r s}^{*} \tilde{p}_{-r,-s}^{*}
$$

Thus, the convolution can efficiently be calculated by performing two inverse Fourier transformations $x^{*} \rightarrow \tilde{x}^{*}, p^{*} \rightarrow \tilde{p}^{*}$ and one forward Fourier transformation $\tilde{q} \rightarrow q$.

\section{References and Notes}

(1) Zander, C., Enderlein, J., Keller, R. A, Eds.; Single-Molecule Detection in Solution - Methods and Applications; Wiley-VCH: Berlin, 2002.

(2) Hofkens, J.; Verheijen, W.; Shulka, R.; De Haen, W.; De Schryver, F. C. Macromolecules 1998, 32, 4493.

(3) Tietz, C.; Jelezko, F.; Gerken, U.; Schuler, S.; Schubert, A.; Rogl, H.; Wrachtrup, J. Biophys. J. 2001, 81, 556.

(4) Deschenes, L. A.; Van den Bout, D. A. Science 2001, 292, 233.

(5) Trabesinger, W.; Renn, A.; Hecht, B.; Wild, U. P.; Montali, A.; Smith, P.; Weder, C. J. Phys. Chem. B 2000, 104, 5221.

(6) Ishijima, A.; Kojima, H.; Higuchi, H.; Harada, Y.; Vale, R.; Funatsu,

T.; Yanagida, T. Biophys. J. 1996, 70, 383.

(7) Kinosita, K. FASEB 1999, 13, S201.

(8) Kinosita, K. Adv. Exp. Med. Biol. 1998, 453, 5.

(9) Kinosita, K.; Itoh, H.; Ishiwata, S.; Hirano, K.; Nishizaka, T. Hayakawa, T. J. Cell. Biol. 1991, 115, 67.

(10) Warshaw, D. M.; Hayes, E.; Gaffney, D.; Lauzon, A. M.; Wu, J. R.; Kennedy, G.; Trybus, K.; Lowey, S.; Berger, C. Proc. Nat. Acad. Sci. 1998, 95, 8034 .

(11) Dickson, R. M.; Norris, D. J.; Moerner, W. E. Phys. Rev. Lett. 1998, 81, 5322 .

(12) Bartko, A. P.; Dickson, R. M. J. Phys. Chem. B 1999, 103, 3053.

(13) Bartko, A. P.; Dickson, R. M. J. Phys. Chem. B 1999, 103, 11237.

(14) Bartko, A. P.; Xu, K.; Dickson, R. M. Phys. Rev. Lett. 2002, 89, 026101 .

(15) Jasny, J.; Sepiol, J. Chem. Phys. Lett. 1997, 273, 439.

(16) Sepiol, J.; Jasny, J.; Keller, J.; Wild, U. P. Chem. Phys. Lett. 1997, $273,444$.

(17) Böhmer, M.; Enderlein J. J. Opt. Soc. B. 2003, 20, 554.

(18) Lieb, M. A.; Zavislan, J. M.; Novotny, L. J. Opt. Soc. Am. B 2004, 21,1210 .

(19) Lukosz, W.; Kunz, R. E. J. Opt. Soc. Am. 1977, 67, 1615

(20) Lukosz, W. J. Opt. Soc. Am. 1979, 69, 1495.

(21) Lukosz, W. J. Opt. Soc. Am. 1981, 71, 744.

(22) Jackson, J. D. Classical Electrodynamics; John Wiley: New York, 1975 .

(23) Török, P.; Higdon, P. D.; Wilson, T. J. Mod. Opt. 1998, 45, 1681.

(24) Enderlein, J. Opt. Lett. 2000, 25, 634.

(25) Enderlein, J.; Böhmer, M. Opt. Lett. 2003, 28, 941.

(26) Abramowitz, M., Stegun, I. A., Eds.; Handbook of mathematical functions; Harry Deutsch: Thun and Frankfurt/Main, 1984. 